\title{
Towards a Blockchain Weather Derivative Financial Instrument for Hedging Volumetric Risks of Solar Power Producers
}

\author{
Olakunle Alao \\ School of Electrical and Electronic Engineering \\ University College Dublin \\ Dublin, Ireland \\ olakunle.alao@ucdconnect.ie
}

\author{
Paul Cuffe \\ School of Electrical and Electronic Engineering \\ University College Dublin \\ Dublin, Ireland \\ paul.cuffe@ucd.ie
}

\begin{abstract}
The weather-dependent nature of solar power makes Solar Power Producers susceptible to the unpredictability of sunshine. Temperature-based weather derivatives have recently emerged as an effective volumetric risk cross-hedge for Solar Power Producers, given that temperature positively correlates with solar radiation. Temperature-based put and call options, mostly traded on organized exchanges, require users to pay premiums that are costly and difficult to price. Swap contracts can serve as an economical alternative since they do not require premiums. However, they are only traded over-the-counter and are thus illiquid and liable to counterparty credit risks. For these reasons, a novel blockchain temperature-based weather derivative swap marketplace is proposed that mitigates the risks inherent in traditional swap contracts. The payoff structure and governing mechanisms of the smart contract that underpins this instrument are also developed. A preliminary investigation of this new financial instrument shows its efficacy in hedging volumetric risks of Solar Power Producers.

Index Terms-Electricity market, renewable electricity, electricity derivatives, decentralized finance, blockchain
\end{abstract}

\section{INTRODUCTION}

Utility-scale Solar Power Producers (SPPS) primarily participate in the pool market for electricity to create revenues to offset their investment costs [1]. These generators are exposed to two different types of volatility risks in this market: price and volume. Price volatility risks arise because of the dynamic nature of electricity markets, which requires that production matches demand at every time $t$, and as such, causes the pool market to clear at different prices from hour to hour [2]. On the other hand, volumetric volatility risks are inherent in SPPs due to their weather-dependent nature, which could make them susceptible to underperform production expectations [3]. Price and volumetric risks together results in volatile cash flows of SPPs that could make obtaining finance from traditional riskaverse sources arduous [4].

Hence, in parallel to the pool market for electricity, SPPs typically resort to price and volumetric risk hedging products in the financial market as in Fig. 1, to stabilize their cash flows

This publication has been funded by the Sustainable Energy Authority of Ireland under the SEAI Research, Development \& Demonstration Funding Programme 2018, grant number 18/RDD/373.

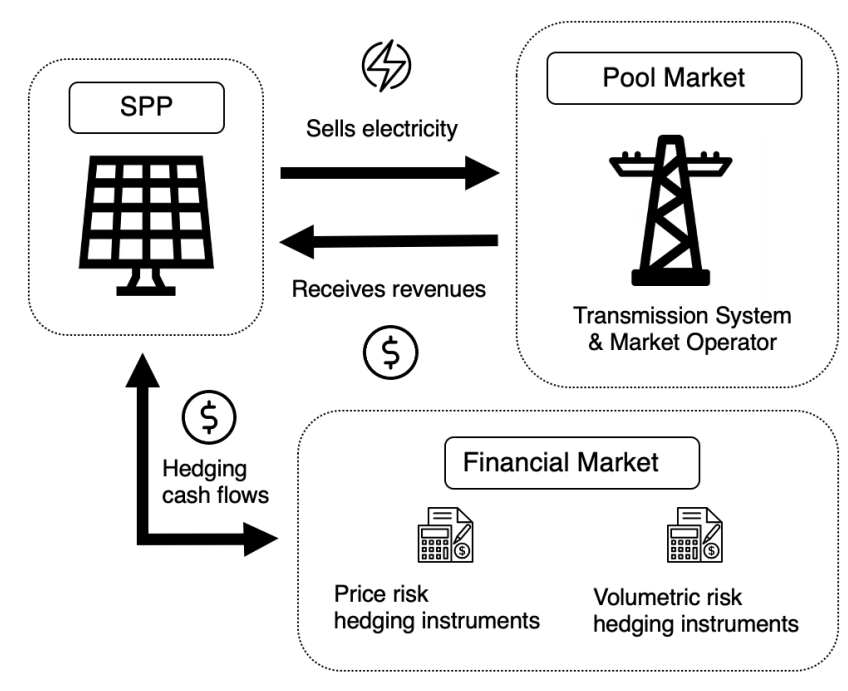

Fig. 1. Demonstration of an SPP trading in the pool market for electricity but simultaneously accessing the financial market to hedge its cash flows

[1]. Price risk hedging instruments are now well understood in comparison to volumetric risk hedging instruments [5].

The volumetric risk of an SPP can be broken down into two categories: short- and long-term. The short-term volumetric risk (i.e., at the delivery period in the pool market) can be mitigated by underbidding or via dispatchable natural gasbased backup generators [3]. The long-term volumetric risk that future generation volumes do not match predicted volumes is much more difficult to ameliorate. Moreover, this risk term has more effect on the SPP's capacity to secure project finance at favorable rates and advantageous terms. The main challenge of an SPP is, therefore, its long-term volumetric risk exposures. Weather derivatives have appeared as a useful tool for mitigating long-term volumetric risks of SPPs because certain weather elements have a positive correlation with SPP electricity generation volumes [6]. 


\section{A. Weather derivatives}

Weather derivatives are financial contracts between bilateral parties whose payoff depends on an underlying meteorological index's evolution during the contract period. The commonly used forms of weather derivatives are call, put, and swap [5].

At the inception of these contracts, contracting parties agree on the weather index (such as the average temperature on a nominated day) that serves as the basis of the contract, a strike value $K$, and a constant $R$ that represents the amount of payment per weather index unit. Call and put options involves a buyer and a seller. At the inception of a call option, the buyer pays the seller a premium. In return, if $\eta$ is greater than $K$ at the maturity date (i.e., the pre-agreed payoff date), the seller pays the buyer an amount equals $R(\eta-K)$ [7]. At the inception of a put option, the buyer also pays the seller a premium. However, the payoff function of a put option differs from a call option. At the maturity date of a put option, the seller pays the buyers an amount equals $R(K-\eta)$ if $\eta$ is less than $K$ [7]. In a swap contract, there is no up-front premium payment, and as such, there is no buying or selling entity [5]. Instead, two parties agree to mutually hedge their weather risks. For instance, the first party can agree to pay its counterparty an amount equals $R(\eta-K)$ if $\eta$ is greater than $K$, and to receive $R(K-\eta)$ if $K$ is greater than $\eta$, or vice versa.

Temperature is currently the most traded weather index [5]. Temperature-based weather derivative contracts are usually traded based on seasonal accumulated heating degree days (HDD) and cooling degree days (CDD). HDD is a measure of the volume of energy required for heating in a day, while CDD is a measure of the volume of energy required for cooling in a day. Buyers enter into these contracts to hedge their weather risk exposures with HDD or CDD as the underlying asset. HDD $h$ and $\mathrm{CDD} c$ are expressed in (1) and (2), respectively. In (1) and (2), $T$ is the number of days over the contract period, $M_{b}$ is the pre-agreed baseline temperature (usually assumed as $18^{\circ} \mathrm{C}$ ), and $M$ is the average of the maximum and minimum temperatures observed for all $t \in T$ [3].

$$
\begin{aligned}
& h=\sum_{t=0}^{T} \max \left(M_{b}-M, 0\right) \\
& c=\sum_{t=0}^{T} \max \left(M-M_{b}, 0\right)
\end{aligned}
$$

Electricity offtakers are historically the most popular users of these indices, given that electricity consumption is significantly affected by seasonal temperature variations [7]. Recently, many temperature-dependent industries, including SPPs are beginning to tap into this relatively new asset class [3], [6]. Temperature-based weather derivatives can serve as an effective volumetric risk cross-hedge for SPPs given the strong positive correlation between solar radiation and temperature [3]. Previously, an increase in temperature spelt a reduced conversion efficiency of solar generators. Recent technological advancements has however mitigated the countereffects of increasing temperature on SPPs' generation volumes [6].

Temperature-based weather derivatives are imperfect hedging instruments since users remain susceptible to the basis risk of temperature not correlating perfectly with the solar radiation that affects production volumes of SPPs. Nonetheless, temperature index is still preferred as the underlying traded weather element for SPPs compared to solar radiation, which perfectly correlates with their output. This choice is because there is an existing liquid pool of players wishing to hedge temperature exposure. And in derivatives, liquidity is crucial since, for every party taking one side, there must be another party willing to take the opposite side. The rest of this paper will be focusing on the use of temperature-based weather derivatives for hedging the volumetric risk of SPPs.

Temperature-based weather derivatives for hedging volumetric risks of SPPs using put and call option contracts have been well studied in [3], [6]. These contracts are available on common and liquid organized exchanges to hedge contracting parties' volumetric risks. The drawback of temperature-based put and call option contracts is the need for users to pay premiums that are typically costly and challenging to price [8]. As a result, SPPs like many other weather-dependent industries, remain reluctant to enter these contracts [8].

We opine that swap contracts with no up-front premium could potentially replace the popular call and put options. A cashflow swap agreement could be feasible, as seen in the following scenario, where the premise is that an abnormally cold season is bad for the SPP [3], [6]. In this condition, if the unusually cold season that is bad for the SPP is at the same time good for another party, it will hence be mutually beneficial for these parties to cooperate in hedging each other's exposure to temperature. However, swap contracts are traded over-the-counter, and as such, are illiquid and liable to counterparty credit risks since bilateral counterparties can fail to fulfil their contractual payment obligations. For these reasons, to date, no study has rigorously analyzed the effectiveness of temperature-based weather derivative swap contracts for hedging volumetric risks of SPPs.

\section{B. Decentralized Finance}

Decentralized Finance (DeFi) instruments are financial services operating on a blockchain [9] - an open, distributed and immutable digital ledger that facilitates secure and transparent transactions between disparate parties [1], [4]. DeFi instruments are underpinned by smart contracts, which are chaincode that self-executes when predefined terms and conditions are met [10], [11].

The concept of DeFi in electricity derivatives is still relatively nascent. The earliest work on DeFi electricity derivative products was undertaken in [12]. In this work, the key regulatory issues regarding the application of blockchain electricity wholesale trading for over-the-counter transactions were studied. Subsequently, more applied forms of the work in [12] have evolved [1], [9]. In [9], the smart contract structure of a blockchain derivative marketplace for hedging electricity price 


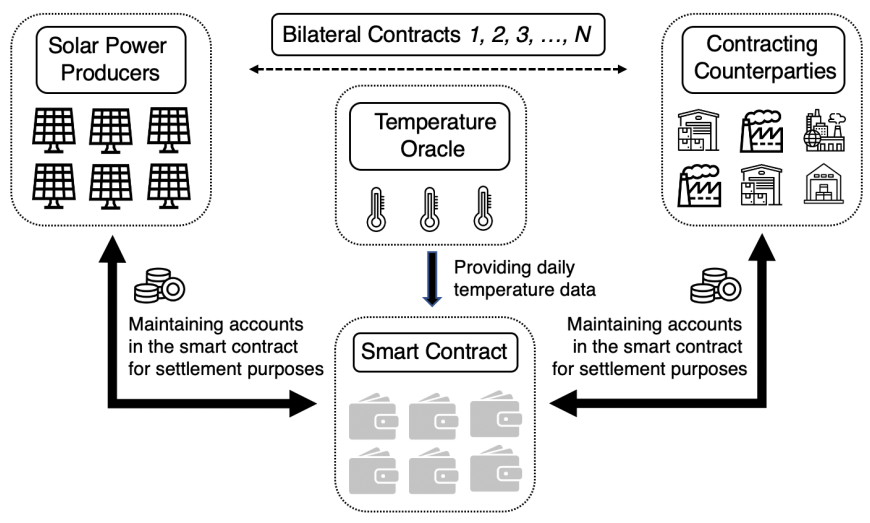

Fig. 2. Schematic of the temperature-based weather derivative swap DeFi instrument

risk of renewable electricity generators has been developed. Results here indicate that DeFi electricity derivative products can significantly reduce hedging costs and mitigate electricity market players' exposure to counterparty credit, margining, legal, process and liquidity risks introduced by traditional electricity price risk hedging instruments [9].

Therefore, it is evident that DeFi concepts can be incorporated into traditional temperature-based weather derivatives swap contracts known to be illiquid and susceptible to counterparty credit risks. Moreover, swap contracts are likely to be attractive to SPPs since they make them bereft of the inefficient task of pricing the usually expensive upfront premium payments in call and put options. The result of the combination of these two concepts is a novel temperaturebased weather derivative DeFi instrument designed to allow mutual hedging between an SPP and a counterparty with an opposite volumetric risk profile to the SPP. In the following sections, preliminary investigations into the effectiveness of this DeFi instrument in hedging contracting parties' volumetric risks is undertaken.

\section{Methodology}

It has already been established that a DeFi swap contract can mitigate the risks introduced by traditional swap contracts. Hence, in this section, the smart contract structure that underpins this DeFi instrument is shown. In a broad sense, the proposed DeFi instrument in Fig. 1 creates a bilateral swap contract marketplace that allows an SPP and another party with an opposite volumetric risk profile to the SPP to mutually hedge each other's volumetric risk.

While the contractual terms between the bilateral contracting parties are written into the smart contract at the inception of their agreement, the smart contract does not naturally have sight of the varying daily temperature published by meteorological entities. Therefore, the smart contract requires a temperature oracle to provide it with the external and varying temperature data [13]. The function of the oracle is to provide the smart contract with the daily temperature data. Hence, the smart contract's three key parties are the SPP, its counterparty and the temperature oracle.

The payoff structure of this novel financial instrument is described in the following sub-sections. We also develop the smart contract governing mechanisms between the bilateral contracting parties that autonomously enforce the proposed payoff structure and ensures that contracting parties remain in the smart contract and act honestly over the specified contract duration.

\section{A. Payoff structure of bilateral contracting parties}

To enable a mutual volumetric risk hedging relationship between an SPP and its counterparty, we propose a swap payoff hedging function for both bilateral contracting parties. This hedging strategy is developed entirely on a blockchain smart contract network and is traded based on a proposed seasonal accumulated deviation degree days (DDD). The DDD tracks the temperature deviation from a specified baseline temperature $M_{b}$. The DDD approach is unique from the traditional HDD and CDD since the specified baseline temperature will depend entirely on the temperature-volume relationship of the contracting parties. The DDD $d$ can be expressed as in (3), where a DDD greater than zero $\left(d^{+}\right)$implies that the temperature in a certain period is warmer than the specified baseline temperature; while, a DDD less than zero $\left(d^{-}\right)$means that the temperature in a certain period is colder than the specified baseline temperature. The temperature index $\eta$ representing the absolute value of the underlying DDD is shown in (4).

$$
\begin{gathered}
d=\sum_{t=0}^{T}\left(M-M_{b}\right) \\
\eta=\left\{\begin{array}{cc}
d & \text { if } d \geq 0\left(d^{+}\right), \\
-d & \text { if } d<0\left(d^{-}\right)
\end{array}\right.
\end{gathered}
$$

An SPP will enter a temperature-based weather derivative swap smart contract with a bilateral counterparty, if it has an opposite volumetric risk profile to the counterparty. At the inception of the contract, the bilateral contracting parties choose an initial DDD hedging position $\left(d_{0}^{-}\right.$or $\left.d_{0}^{+}\right)$. The initial DDD hedging position of a contracting party that intends to hedge against temperatures below a specified baseline temperature will be $d_{0}^{-}$. Conversely, the initial DDD hedging position of a contracting party that intends to hedge against temperatures above a specified baseline temperature will be $d_{0}^{+}$. In both cases, the bilateral counterparty must take an opposite initial DDD hedging position.

A contracting party receives a payoff $\Phi_{\text {swap }}$ from its bilateral counterparty as in (5), if the DDD on the actual day is in the same direction on the number line as its initial DDD hedging position (see Fig. 3). That is, if on the actual day, the DDD is equivalent to $d^{+}$and the contracting party holds an initial DDD hedging position of $d_{0}^{+}$, or the DDD on the actual day is $d^{-}$and the contracting party holds an initial DDD hedging position of $d_{0}^{-}$. On the other hand, the contracting party returns a payoff to its bilateral counterparty as in (5), if 


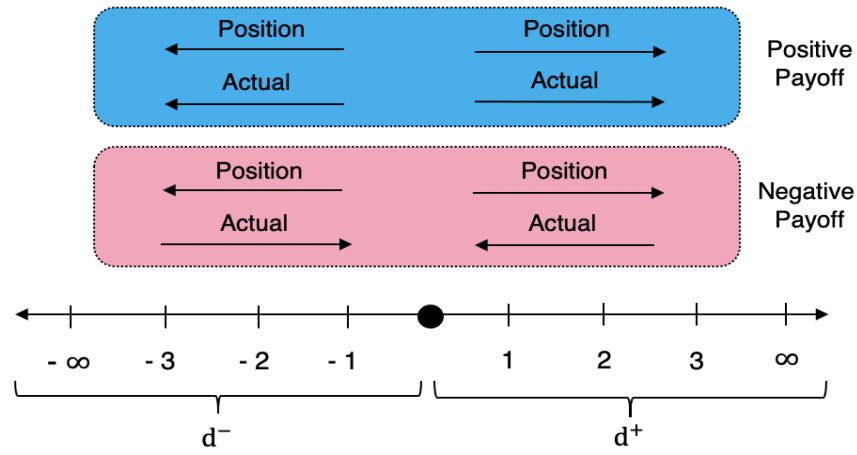

Fig. 3. DDD's number line depicting the payoff of contracting parties on the actual day based on their initial DDD hedging position

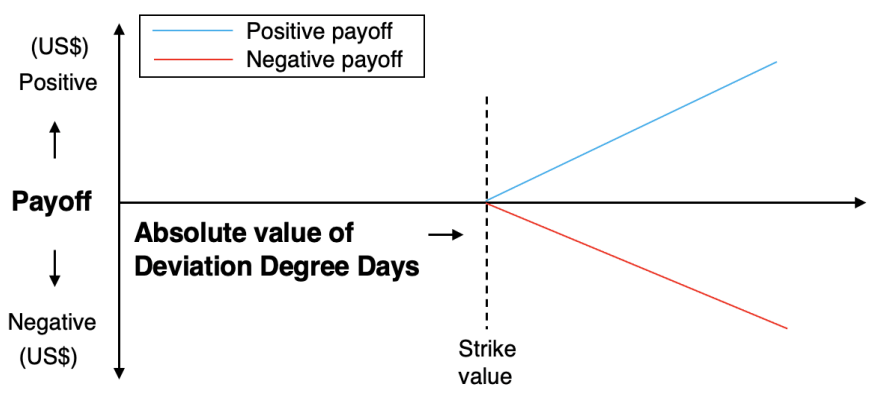

Fig. 4. Payoff diagram of proposed temperature-based weather derivative swap DeFi smart contract

the DDD on the actual day is in a different direction on the number line as its initial DDD hedging position (see Fig. 3). That is, if on the actual day, the DDD is equivalent to $d^{+}$and the contracting party holds an initial DDD hedging position of $d_{0}^{-}$or the DDD on the actual day is $d^{-}$and the contracting party holds an initial DDD hedging position of $d_{0}^{+}$. In (5), $R$ is the US\$ payment per degree day and $\eta$ is the temperature index.

$$
\Phi_{\text {swap }}=R \times \max (\eta-K, 0)
$$

Fig. 3 shows how the initial DDD hedging position of contracting parties affect their payoff. Fig. 4 displays the payoff diagram of the bilateral contracting parties, either receiving a positive payoff or incurring a negative payoff.

\section{B. Smart contract governing mechanisms between bilateral contracting parties}

1) Settlement mechanism: The payoff settlement-related risk exposures of contracting parties in a traditional overthe-counter derivative contract are margining risks due to the infrequent settlement times of the broker that clears and settles transactions [5]; third-party risks arising from the probability that the broker can become fraudulent or insolvent [14]; and legal risks due to possible contractual disputes between contracting parties [2]. In earlier work in [9], we have established that a payoff settlement mechanism enforced by a DeFi derivative smart contract can ameliorate these risks. The

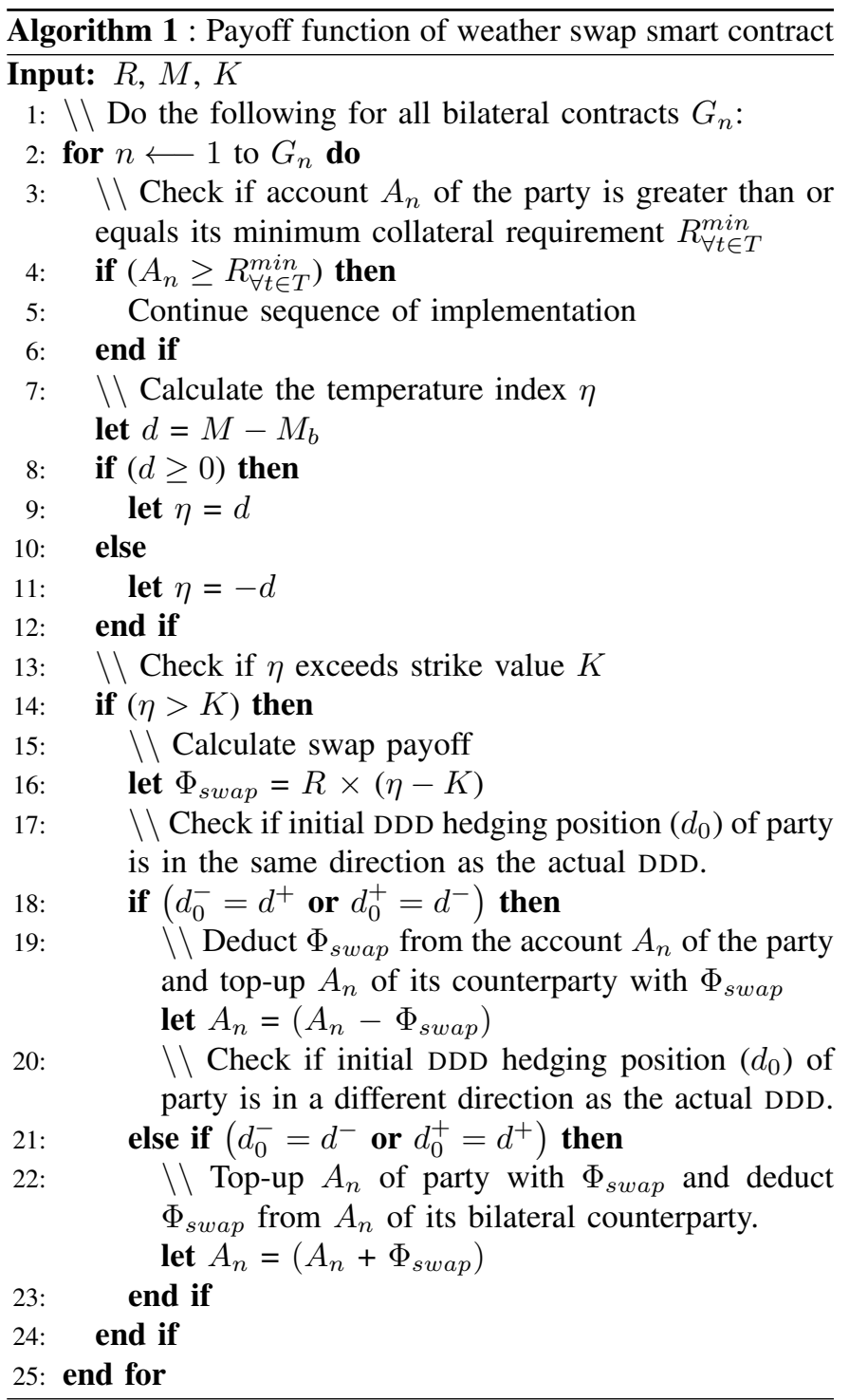

sequence of execution of the payoff settlement mechanism of the proposed temperature-based weather derivative swap DeFi smart contract between SPPs and their bilateral counterparties is shown in Algorithm 1. This chaincode irrefutably executes once the smart contract is deployed on the blockchain.

2) Incentive mechanism: Counterparty credit risk, the risk that a contracting party can incur financial losses due to a default on settlement payment by its bilateral counterparty [15], is the main risk of contracting parties in an over-thecounter derivative transaction [16]. Again, in [9], we have shown that a well-designed incentive mechanism built into a DeFi derivative smart contract can reduce counterparty credit risk on-chain and in a fully autonomous way. In this schema, collaterals are autonomously held and managed by the smart contract on behalf of contracting parties for settlement purposes, unlike in traditional over-the-counter contracts where collateral is managed by third-party trusted brokers [9].

These collaterals must be adequate to incentivize rationality amongst contracting parties [9]. A collateral within a DeFi 
derivative smart contract constitutes a maintenance margin and termination penalty component [9]. Maintenance margins are reserved to guarantee that contracting parties have adequate funds in their accounts to make settlement payments due to their bilateral counterparty [9]. The minimum maintenance margin $E_{\forall t \in T}^{\min }$ that must be reserved by all contracting parties in the smart contract is represented in (6). The maximum payoff $\Phi_{\text {swap }}^{\max }$ that can be due to any contracting party is shown in (7).

$$
\begin{gathered}
E_{\forall t \in T}^{\min } \geq \Phi_{\text {swap }}^{\max } \\
\Phi_{\text {swap }}^{\max }=\underset{R, \eta}{\operatorname{argmax}}\left(\Phi_{\text {swap }}\right)
\end{gathered}
$$

On the other hand, the termination penalty $N$ component of the collateral is crucial in discouraging contracting parties from prematurely exiting the contract. In practice, the termination penalty should cover the replacement cost of the contract that will be incurred by the non-exiting contracting party [5]. Contracting parties have the liberty to specify a suitable termination penalty between them and their bilateral counterparties [9]. Therefore, the minimum collateral requirement $R_{\forall t \in T}^{\min }$ of each contracting party in the smart contract is the sum of its maintenance margin and termination penalty as in (8) [9]. Contracting parties whose smart contract account falls below their minimum collateral requirement will lose their termination penalty deposit and, thereafter, have their accounts immediately liquidated by the smart contract.

$$
R_{\forall t \in T}^{\min }=E_{\forall t \in T}^{\min }+N
$$

\section{TEST PLATFORM}

This section describes the test platform used to demonstrate the functioning and value of the proposed temperature-based weather derivative swap DeFi instrument.

\section{A. Simulation of Temperature and SPP's generation profile}

Temperature is one of the key weather elements that affect the generation volumes of an SPP [3]. Therefore, the prediction of temperature is of interest to an SPP because temperature has a positive correlation with solar radiation and thus solar power generation [6]. In Fig. 5, a notional temperature profile using the model in [6] is shown. Here, the actual temperature in a winter month of December is presumed to be much colder than predicted. Given the temperature-SPP generation relationship, the electricity generated by the SPP can be approximated as an affine function of temperature (see Fig. 6), where the variable term of the function is arbitrary. The investigation of the robustness of the temperature and SPP generation models employed here is beyond the scope of this work.

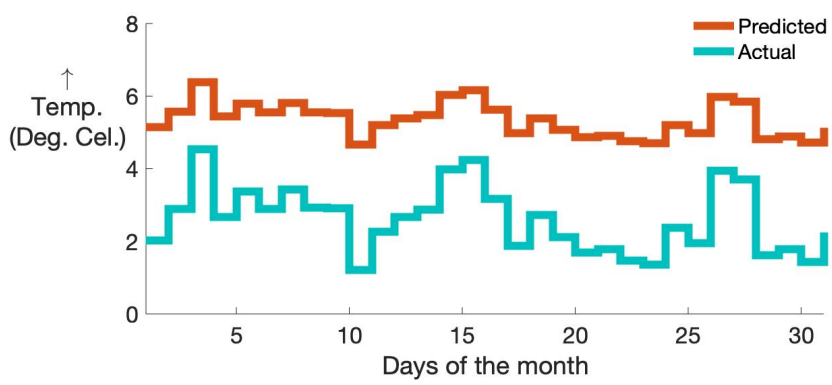

Fig. 5. Predicted and actual temperature in a winter month of December

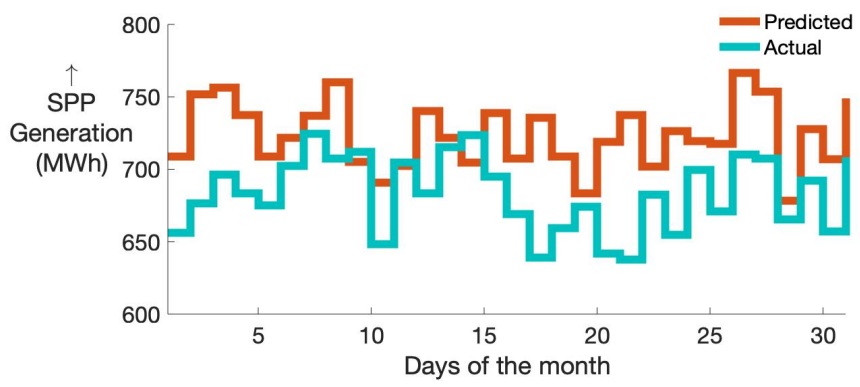

Fig. 6. Predicted and actual electricity generation profile of an SPP with a 300MW installed capacity in a winter month of December

\section{B. Electricity Market Assumptions}

The following assumptions are made in the estimation of the SPP's cash flow:

- The costs incurred due to the short-term deviation between the offered and actual electricity generated to the pool market at the delivery time is ignored.

- The Market Operator of the pool market accepts all the electricity offered by the SPP at every trading period.

- The volatile electricity prices of the pool market has been hedged.

\section{DeFi Instrument Assumptions}

The following assumptions are made in the design of the DeFi instrument:

- The native currencies of blockchain technologies are highly volatile, which could expose contracting parties in a smart contract to the possibilities of incurring financial losses. To hedge against this volatility risk, a stablecoin, as in [17] is incorporated into the smart contract [9]. The stablecoin is pegged to the fiat currency that the pool market for electricity is denominated in (i.e., 1 stablecoin equals US\$1).

- While the smart contract can read and react to the data provided by the temperature oracle, fraudulent actors can manipulate this data stream to alter the actions of the smart contract [9]. To mitigate the security risks posed by these fraudulent actors, a decentralized oracle, as in [18] is designated to act as a trustworthy temperature oracle in the smart contract. 


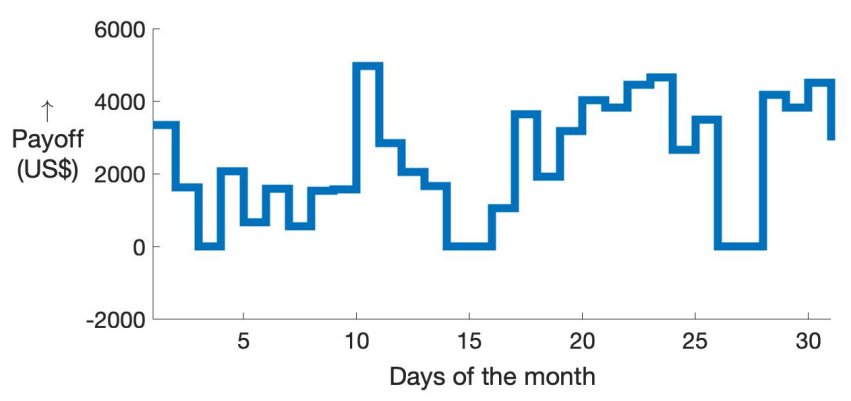

Fig. 7. Payoff profile of the SPP autonomously enforced by the smart contract

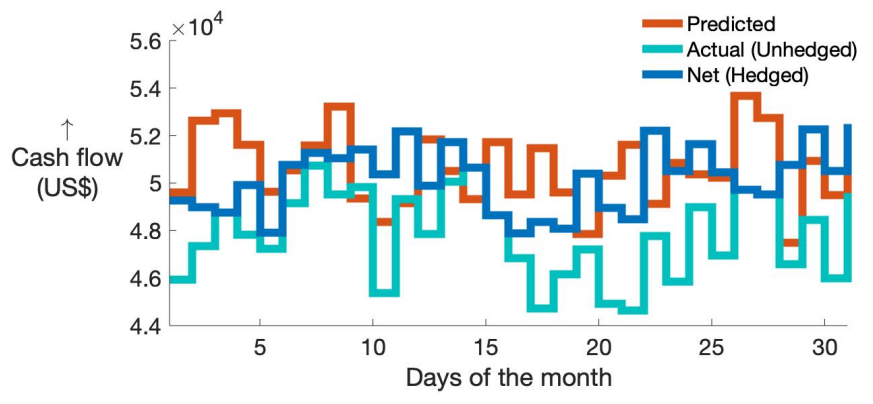

Fig. 8. Predicted, actual and net cash flow analysis of the SPP

\section{REsults}

The inherent volumetric risk exposures of weatherdependent industries to the vagaries of weather necessitate hedging, as established in Section I. This section demonstrates how the proposed temperature-based weather derivative swap DeFi instrument mitigates the volumetric risk exposure of an SPP.

Consider a 300MW SPP in the winter month of December whose generation profile is as in Fig. 6. In this scenario, it is evident that the actual generated electricity is suppressed due to a much colder than predicted winter month of December (see Fig. 5). Fortunately, prior to the winter month of December, the SPP and a beverage company had enrolled in the proposed DeFi instrument to mutually hedge against their volumetric risks in December with DDD as the underlying asset. The rationale for this agreement was for the SPP to hedge against low generation volumes due to an abnormally cold winter (i.e., an initial position: $d_{0}^{-}$) and for the beverage company to hedge against low volume of sales due to an abnormally warm winter (i.e., an initial position: $d_{0}^{+}$).

The other terms of their agreement include a daily maturity or payoff settlement (i.e., $T=1$ ), a baseline temperature of $4.7^{\circ} \mathrm{C}$, a strike value per day of $1 \mathrm{DD}$, and a US $\$$ payment per degree day of US $\$ 2000$. With these terms, the payoff due to the SPP becomes as in Fig. 7. If all the electricity generated by the SPP is sold to the pool market at US\$70/MWh, then its predicted, actual, and net cash flows will be as in Fig. 8. It is apparent that by enrolling in the proposed DeFi instrument, the SPP has stabilized its cashflows (i.e., net) and has reduced the financial losses it could have incurred due to the suppressed generation volumes caused by an abnormal weather condition.

\section{CONClusion}

This paper has shown that a temperature-based weather derivative swap DeFi instrument can serve as an effective volumetric risk hedging instrument for an SPP and at a negligible cost. The proposed derivative instrument is only elucidative of the type of flexible hedging arrangements that can be enabled by blockchain, as any other weather index could be employed if required. Future work will involve developing a robust temperature-solar model; and a rigorous assessment of the proposed DeFi instrument against other weather derivative instruments, and across different seasons, hedging positions, and risk metrics.

\section{REFERENCES}

[1] O. Alao and P. Cuffe, "Towards a blockchain contract-for-difference financial instrument for hedging renewable electricity transactions," in 2020 6th IEEE International Energy Conference (ENERGYCon), 2020, pp. 858-863. DOI: 10.1109/ENERGYCon48941.2020.9236436.

[2] Min Liu, F. F. Wu, and Yixin Ni, "A survey on risk management in electricity markets," in 2006 IEEE Power Engineering Society General Meeting, 2006.

[3] S. Bhattacharya, A. Gupta, K. Kar, and A. Owusu, "Cross Hedging Strategies for Solar Energy Production using Weather Derivatives," Ssrn, no. 1408333 , pp. 1190-1195, 2015. DOI: 10.2139/ssrn.2642685.

[4] O. Alao and P. Cuffe, "Towards a blockchain special purpose vehicle for financing independent renewable electricity projects in sub-saharan africa," in 2020 6th IEEE International Energy Conference (ENERGYCon), 2020, pp. 1041-1046. DOI: 10.1109/ENERGYCon48941.2020. 9236599.

[5] J. Hull, Options, Futures and Other Derivatives. 2012, ISBN: 0135009944. DOI: 10.1007/978-1-4419-9230-7_2.

[6] T. Kanamura, "Volumetric risk hedging strategies and basis risk premium for solar power," Journal of Alternative Investments, vol. 23, no. 1, pp. 35-47, 2020, ISSN: 15203255.

[7] L. Zeng, "Weather derivatives and weather insurance: Concept, application, and analysis," Bulletin of the American Meteorological Society, vol. 81 , no. 9, pp. 2075-2082, 2000, ISSN: 00030007. DOI: 10.1175/ 1520-0477(2000)081<2075:WDAWIC $>2.3 . C O ; 2$.

[8] H. Hamisultane, "Which Method for Pricing Weather Derivatives ? To cite this version : HAL Id : halshs-00355856 Which Method for Pricing Weather Derivatives ?," 2009.

[9] O. Alao and P. Cuffe, "Structuring Contract-for-Difference Instruments for Hedging Electricity Price Risks on a Blockchain-based Marketplace," in IEEE TechRxiv, 2020, pp. 1-12.

[10] K. Christidis and M. Devetsikiotis, "Blockchains and Smart Contracts for the Internet of Things," IEEE Access, vol. 4, pp. 2292-2303, 2016, ISSN: 21693536. DOI: 10.1109/ACCESS.2016.2566339.

[11] A. de Villiers and P. Cuffe, "A three-tier framework for understanding disruption trajectories for blockchain in the electricity industry," IEEE Access, vol. 8, pp. 65 670-65 682, 2020.

[12] C. I. Dick and A. Praktiknjo, "Blockchain technology and electricity wholesale markets: Expert insights on potentials and challenges for OTC trading in Europe," Energies, vol. 12, no. 5, 2019, ISSN: 19961073. DOI: 10.3390/en12050832.

[13] J. Adler and et al., "ASTRAEA: A Decentralized Blockchain Oracle," in 2018 IEEE International Conference on iThings, GreenCom, CPSCom and SmartData, 2018, pp. 1349-1354, ISBN: 9781538679753. DOI: 10. 1109/Cybermatics.

[14] S. J. Deng and S. S. Oren, "Electricity derivatives and risk management," Energy, vol. 31, no. 6-7, pp. 940-953, 2006, ISSN: 03605442. DOI: 10.1016/j.energy.2005.02.015.

[15] M. Morini, "Managing Derivatives on a Blockchain. A Financial Market Professional Implementation," SSRN Electronic Journal, 2017.

[16] C. P. Fries and P. Kohl-landgraf, "Smart Derivative Contracts: Detaching Transactions from Counterparty Credit Risk," pp. 1-22, 2018.

[17] Maker Team, "The Dai Stablecoin System," Tech. Rep., 2017.

[18] J. Peterson, J. Krug, M. Zoltu, A. K. Williams, and S. Alexander, "Augur: a decentralized oracle and prediction market platform," pp. 1-16, 2015. DOI: 10.13140/2.1.1431.4563. arXiv: 1501.01042. 